\title{
Sign Gesture Recognition Using Modified Region Growing Algorithm and Adaptive Genetic Fuzzy Classifier
}

\author{
Rajesh Kaluri $^{1 *} \quad$ Pradeep Reddy $\mathrm{Ch}^{1}$ \\ ${ }^{1}$ School of Information Technology and Engineering, VIT University, Vellore, India. \\ * Corresponding author’s Email: rajesh.kaluri@vit.ac.in
}

\begin{abstract}
Sign language is the most communal language used by the deaf people among themselves for any kind of communication. People who are not familiar with Sign Language face difficulty in interacting with the deaf people. Hence, an effective system should be established to attain and discriminate the sign language. In our proposed system, we developed a framework for extracting and recognizing the sign gesture language and which is classified into four stages like noise removal using median filter, Segmentation using Modified Region Growing Algorithm (MRGA), feature extraction and recognition using Adaptive Genetic Fuzzy Classifier (AGFC). We have used Genetic algorithm with Fuzzy classifier to find out the optimal rules generated by Fuzzy classifier.
\end{abstract}

Keywords: Adaptive Genetic Fuzzy Classifier, Gesture recognition, Region Growing algorithm, Sign Language Recognition.

\section{Introduction}

The process of identifying and classifying significant activities by individual's hands, arms, face, and at times head is identified as Gesture recognition. It is an imminent field of study because of its implication in planning affectedly intellectual human-computer edges for several presentations. They extent from sign language through medical analysis to effective certainty [1]. Human-computer communication tools steadily create its mode as the supreme widely held study theme because of the fast growth of computer knowledge. In addition, the significance of sign points out its assessment in the field of investigation of human-computer communication. Meanwhile signal is the most usual, instinctive and informal to study resources of human-computer communication it is extensively investigated and practiced. Gesture recognition centered on visualization also has turn into one of the broadly exploration subject [2]. Communication via gestures as a sort of signal is a standout amongst the most regular strategies for exchanging information for most deaf people. The target of communication via gestures acknowledgment (SLR) is to provide a powerful and impeccable mechanism to record gesture based communication into words or speech so that collaboration amongst deaf and other mankind can be more reasonable. SLR, as one of the imperative research ranges of humancomputer Interaction (HCI), has brought forth more enthusiasm for an HCI society. [3].

The fast progress of computer technology has directed to the development of human computer interaction (HCI) methods. Lately, a very attractive substitute, a movable method set in with inertial devices was recommended to feel the doings of humans. This was used to seizure his/her gesture path info from speeding up for distinguishing signals or writing [4]. When a similarity is depicted with former skills which used some wearable measuring device or pointers, marker-less visionbased methods offers more ordinary and less invasive resolutions. They are more suitable for realworld placements $[5,6]$. Locally unrelated languages have been established such as American 
Sign Language (ASL) and German Sign Language [7]. Hand structure and gesture are separated certainly and exactly using these devices. Though, devices are exclusive and, remarkably, they decrease the spontaneity of sign language communication [8, 9]. Gesture recognition relates to identifying expressive terms of gesture by a human, comprising the hands, arms, face, head, and/or body. It is of greatest significance in planning a quick and wellorganized human-computer interface. The submissions of gesture recognition are assorted, varying from sign language through medical restoration to effective realism $[10,11]$.

The principle goal of motion acknowledgment is delivering a framework which distinguishes distinct human motions in that way utilizing them to transport data or for the limitation of the gadget [12]. Gesture recognition is the course of realizing and organizing expressive actions by a human's hands, arms, face, and at times head $[13,14]$. Recognition of the launching opening and ending of a definite gesture from an endless trail gesture arrangement has won a lot of acceptance in the previous ages [15, $16,25]$.

The proposed method gives better results in terms of classifying the textures and we used Modified Region Growing algorithm for efficient segmentation process. AGFC and Adaptive Genetic Algorithm helped in optimizing selection rules whereas conventional Neural Networks method was lagging in finding the gestures of the shaded images.

The rest of the paper is structured as Unit II gives the literature review of the existing methods. Unit III displays our projected process being used for acknowledgement of sign gesture using adapted section developing and AGFC. Unit IV elucidates the effect of the projected method and in conclusion Unit $\mathrm{V}$ gives our projected technique with proposals given for forthcoming mechanisms.

\section{Literature Review}

Sign language creates an imperative transmission network among the deaf. Kosmidou et al. [17, 18] have projected improved SL acknowledgement by linking the discrete way of contracting with the signer's level of deafness through a hybrid adaptive weighting method used to shallow electromyogram and 3-D accelerometer data. However, they are unsuccessful in showing the better results for the extension of IMEn analysis for both the hands.

Tubaiz et al. [19] have anticipated a glove-based Arabic sign language appreciation system using a method for progressive data arrangement. In the dataset, hand actions are seized using two DG5VHand data gloves. Data cataloguing was accomplished using a camera to coordinate hand activities with their consistent sign language words. But, the system cannot be efficiently used to classify and recognize the objects which are distant from the camera.

Along with Kinect depth camera, Yuan Yao et al. [20] displayed a new super pixel-based hand motion detection method. This growth was founded on an original super pixel earth mover's space metric. So as to create marker less hand origin, effective application of the complexity and minimum evidence from Kinect was prepared. Authors failed to show the better accuracy for the various positions of hand and also their method is limited in classification for the hand parts.

Yang et al. [21] have deliberated two vital complications in endless sign language appreciation from unassisted video preparations. At the sentence level, they deliberated the movement epenthesis difficulty and at the feature level, the problem of hand separation and alignment. However, the algorithm is not applicable to all the general motion patterns of a human and also for all the continuous sign sentences.

Li et al. [22] have offered an acknowledgment system for admitting the verses of home-serviceassociated sign language. As the data established from a sensor are in sequence and the hidden Markov models (HMM) that have been magnificently operated to speech signals was selected as a classifier. To elucidate this tricky, an entropy-based $\mathrm{K}$-means algorithm was projected to assess the number of conditions in the HMM model with an entropy illustration. Four actual datasets are developed to prove the established entropy-based $\mathrm{K}$ -means algorithm. In addition, a data-driven technique was specified to syndicate the simulated bee colony algorithm with the Baum-Welch algorithm to regulate the arrangement of HMM. Nevertheless, the system is not able to find the exact number of clusters for the dataset used and also failed to recognize all the signs (dataset) of human efficiently. 
Ahmed [23] have introduced a real-time hand gesture recognition system. The technique is divided into three phases like image acquisition, feature extraction, and recognition. Initially input image of hand gestures are captured using a digital camera in fairly accurate frame rate. Next rotation, translation, scaling and orientation invariant feature extraction methods have been initiated to mine the feature of the input image based on moment feature extraction method. Finally, a neural network was used to recognize the hand gestures. However, the method used in the paper failed to extract the features under dark portions and also not showed the better results in recognizing the gestures of a human.

\section{Proposed Methodology}

Sign language is the common language used by the deaf people for communication. Even though they appropriately converse with each other by using Sign Language, they confront many problems when they attempt to converse with folks who can listen to, particularly those who are not aware in Sign Language it is like recognizing the presence of an unseen communication barrage. Therefore, an effective technique would be established to attain and identify the sign gesture languages. In our proposed system, we framed a method for examining and identifying the sign gesture language. Our proposed method targets at connecting transmission interludes among the deaf group and other people. The pictorial representation of steps included in our sign gesture recognition is revealed in Fig 1.

\subsection{Noise removal using Median filter}

Noise removal is a significant process in image processing and we used median filter for noise removal. The median filter is frequently employed to overcast worth images due to its belongings of control protective flattening. In the median filtering operation, the pixel values in the neighbourhood window are graded according to intensity, and the median value turn out to be the output for the pixel under evaluation. The succeeding stages were accustomed to eliminate the noise from images.

In median filtering, the adjacent pixels are graded in line with illumination and the average value turn out to be the new central pixel value. Median filter ensures brilliant work of rejecting particular forms of noise, in actual, "shot" or impulse noise in which some individual pixels have higher values. The common countenance for the median filter is assumed as per the eq1.

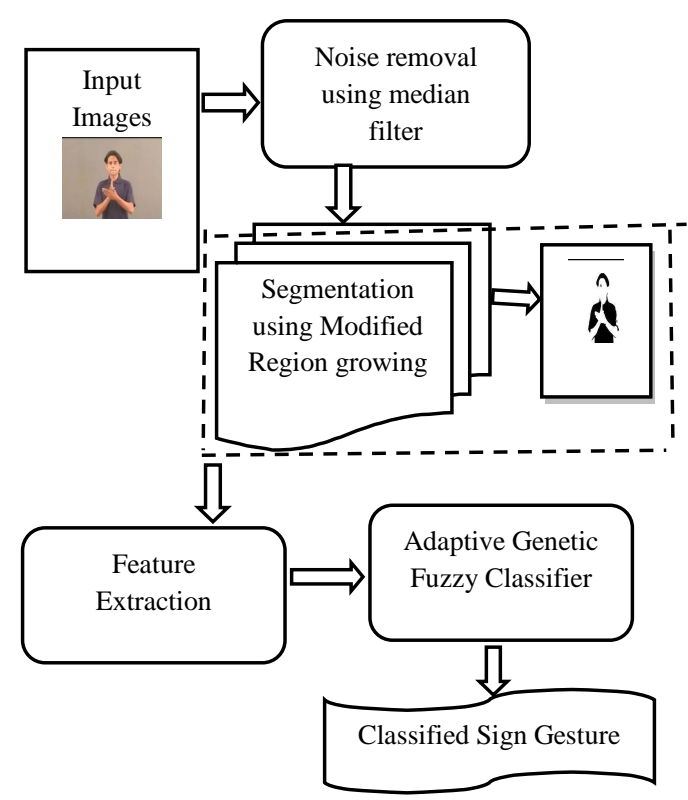

Figure.1 Flow diagram for Proposed Sign Gesture Recognition.

$$
M f_{0}\left(p_{1}, p_{2} \ldots p_{n}\right)=\operatorname{MIN}\left(\sum_{k=1}^{n}|| p_{1}-p_{k} \mid \ldots \sum_{k=1}^{n}\left\|a_{n}-a_{k}\right\|\right.
$$

Where $M f_{o}$ - Median Filtered output,

$p_{1}, p_{2} \ldots p_{n}$ - number of pixels under evaluation

$n$ - number of pixels

With Eq. 1, the median filtering is executed to eliminate the noise from the developed image. After the noise elimination is completed, the subsequent step in our projected technique is the segmentation method.

\subsection{Image Segmentation using Modified Region Growing}

Segmentation is a technique utilized in image processing in order to extract particular regions which are easier for analysis. Various segmentation algorithms exist like watershed algorithm, K-means algorithm, level set algorithm, clustering algorithm etc. In our proposed system, we have utilized modified region growing algorithm for segmenting the gesture from frames.

The region growing segmentation comes under the pixel based segmentation method since the major consideration here is pixels in an image. Initially the seed points are randomly chosen and the neighbouring pixels surrounding this seed point are inspected to conclude whether these pixel neighbours should be added to the particular region or not. The process is repeated in order to find out different regions in the image. The region growing segmentation utilizes "intensity" constrain for growing a region based on seed pixel. The major 
disadvantage of these processes lies in selection of seed point.

The modified region growing segmentation method can be divided into three phases like gridding, seed point selection and growing the region around the selected pixels. The gridding is usually a image division process where we divide the particular image into different grids which are square in shape. In our proposed technique, we split the image into 4, 18 and 24 grids. For each of the grid image, the region is grown based on the selected seed point. The histogram for every pixel in the grid is found out and the seed points are selected based on this histogram analysis. The image value ranges from 0 to 255 since it is a grey scale image. For each grid, frequent histogram value is selected as the seed point pixel. From this we chose any one of the pixel as seed point for the particular grid.

Once the seed point is estimated, the region is grown from it by comparing the seed point with the neighbouring pixels. The region is grown with the selected neighbouring pixel only if the pixel satisfies the condition else it will be neglected from the region. As mentioned earlier we utilize modified region growing for segmentation where the constraints are "intensity" and the "orientation" where as in normal region growing the intensity constrains alone is used for region growing. For calculating both intensity and orientation we need to set particular conditions. For the intensity constraint, in-order to check if the neighbour pixel satisfies the required condition, an intensity threshold is assigned. The difference between the neighbour pixel and the pixel under consideration are estimated and the maximum difference value is selected as the threshold.

Let the intensity value of the pixel be $P_{i}$ and that of neighbouring pixel is $Q_{i}$. The intensity threshold is set to be $I_{t h}$, then

$$
\left\|P_{i}-Q_{i}\right\| \leq I_{t h}
$$

If the above condition is satisfied, then the intensity constraint is selected.

For estimating the "orientation" constraint, the $X$ and $Y$ axis gradients are found out. Let $H_{x}$ be image values after applying the gradient in $X$ axis and $H_{y}$ be the values after applying gradient in $Y$ axis. Once the gradient is found out, the pixel values which had a value from 0 to 255 is transformed to 0 to 3.14 . The gradient values in radian are converted into degree which ranges from 0 to 180 degrees and gives the orientation values. Then both the values are combined to form the gradient matrix $G$ where $G$ $=1 / 1+\left(H_{x}^{2}+H_{y}^{2}\right)$.
From the matrix $G$, we can get the orientation of each of the pixels. Let the orientation value of the pixel be $P_{o}$ and that of neighbouring pixel is $Q_{o}$. The orientation threshold is set to be $O_{t h}$ then

$$
\left\|P_{o}-Q_{o}\right\| \leq O_{t h}
$$

If the above condition is satisfied, then the orientation constraint is selected. The region is grown to the neighbouring pixels which satisfy the conditions of intensity constraint and the orientation constraint. For every grid, the region is grown and based on these regions features are extracted.

\subsection{Feature Extraction}

Once the image segmentation using Modified Region Growing Algorithm is done, the next phase in our proposed method is the feature extraction where various features for processing the image are extracted. Here the feature vectors containing information about the aspect ratio, holo entropy, texture based features are extracted.

3.3.1 Aspect Ratio: The ratio between length and the width of the particular pixel in the images is defined as aspect ratio which is given by the expression

where,

$$
A_{r}=L / W
$$

$L$ - Pixel length; $W$ - Pixel width.

3.3.2 Holo Entropy: Holo entropy is an arithmetical measure of unpredictability that can be used to distinguish the texture of the input image. The holo entropy basically depends on the histogram value in the image. The holo entropy can be measured in terms of the summation of entropies on all the attributes in the particular section of the image.

Holo Entropy can be represented in terms of summation of entropies which is shown below

$$
\mathrm{EN}_{\mathrm{hl}}(\mathrm{Z})=\sum_{\mathrm{i}=1}^{\mathrm{n}} \mathrm{E}\left(\mathrm{Z}_{\mathrm{i}}\right)
$$

Where $E N_{h l}(Z)$ - Holo entropy for the image $Z$ $E\left(Z_{i}\right)$ - Entropy of image pixel.

\subsubsection{Texture Feature Extraction using Gabor Wavelet Transform:}

In feature extraction, texture feature holds an important role as it is an extensive feature in an image. Even specific feature in an image can be easily extracted by utilizing a texture feature. The texture features can be applied for shape computation, segmentation, recognition etc. The primitive and placement rules are necessary in order to extract the texture from images. In our proposed technique, the texture feature is extracted using 
Gabor filters. The spatial domain expression for Gabor filter is given in the equation below

$G f_{\sigma, \emptyset, \theta}(p, q)=G_{\sigma}(p, q) \cdot \exp [2 \pi k \emptyset(p \cos \theta+q \sin \theta)]$

Where

$G_{\sigma}=\frac{1}{2 \pi \sigma^{2}} \exp \left[\left(p^{2}+q^{2}\right) / 2 \sigma^{2}\right]$

$\emptyset$ - Frequency of the span-limited sinusoidal grating

$G_{\sigma}(p, q)$ - Gaussian function

$\sigma$ - scale parameter

$\theta$ - Orientation in the interval $[0,1]$

$p, q$-coordinates of an image

The Gabor filter is a complex valued function and it is decomposed into real and imaginary parts as

$$
G f_{\sigma, \emptyset, \theta}(p, q)=R E_{\sigma, \varnothing, \theta}(p, q)+j \operatorname{Im}_{\sigma, \varnothing, \theta}(p, q)
$$

Where

$R E_{\sigma, \emptyset, \theta}(p, q)=G_{\sigma}(p, q) \cos [2 \pi \emptyset(p \cos \theta+q \sin \theta)]$ represents real part

$\operatorname{Im}_{\sigma, \emptyset, \theta}(p, q)=G_{\sigma}(p, q) \sin [2 \pi \emptyset(p \cos \theta+q \sin \theta)]$ represents imaginary part

The image with Gabor function is subjected to convolution to obtain Gabor-filtered output. The convolution of image $I(p, q)$ corresponding to real and imaginary components of Gabor filter for a neighbourhood window $Z x Z$ is given as

$$
\begin{gathered}
C n_{R E}(p, q / \sigma, \emptyset, \theta)=\sum_{\mu=1}^{i} \sum_{t=1}^{i} I(p+\mu, q+ \\
t) R E_{\sigma, \varnothing, \theta}(\mu, t) \\
C n_{I m}(p, q / \sigma, \emptyset, \theta)=\sum_{\mu=1}^{i} \sum_{t=1}^{i} I(p+\mu, q+ \\
t) I_{\sigma, \emptyset, \theta}(\mu, t)
\end{gathered}
$$

In conventional Gabor filter design the better filter parameter are chosen so that the texture extracted will be accurate with maximum related energy. The texture thus obtained using the Gabor filter contain even a minute feature values in the image. Once these features are extracted the final stage is the classification process. In the proposed method, the classifier utilized is the Adaptive genetic fuzzy classifier (AGFC).

\subsection{Classification using AGFC}

The Adaptive Genetic Fuzzy classifier is the modified form of fuzzy classifier where we utilize the adaptive genetic algorithm for the optimization of the fuzzy rules. The classifier provides exact classification of sign gesture which aid in improved recognition rate. The fuzzy classifier and genetic algorithm operation are explained in the section below

\subsubsection{Fuzzy Logic}

Fuzzy logic is generally a method based on fuzzy set theory, which can be used to solve multifaceted problems. The fuzzy logic operates based on three phases such as
a. Fuzzification
b. Inference
c. Defuzzification

Fuzzification is the process where the input feature vectors are converted into fuzzy values with the aid of fuzzy membership function which is used to correlate with the score of each fuzzy value. The second phase is the fuzzy inference engine where the mapping between input and output membership function is done based on the fuzzy rules [26]. Defuzzification is the final phase in fuzzy logic which combines the output into a single numerical value.

Fuzzy Triangular Membership Function: The fuzzy triangular membership function is used to convert the numerical feature vector values into fuzzy numbers. The selection of membership function is done using various machine learning methods like artificial neural networks, genetic algorithms etc or it can be a random value. The membership function comes in different shapes such as; triangular, trapezoidal, piecewise-linear, Gaussian, bell-shaped, etc. The major consideration in fuzzy logic is the selection of membership function. The selection is done based on the problem type or its size. The entire fuzzy set has to be completely defined based on the membership function. The appropriate membership function that we select must possess the above condition.

In our proposed system, we have chosen triangular membership function for fuzzy classification since it provides better membership value based on three feature vector space. As the name suggests, the triangular membership function consists of three vertices $a, b$ and $c$ for $x$ coordinates of $f(x)$ in a fuzzy set. Here a represents the lower boundary and $c$ is the higher boundary and $b$ is the centre. The lower and the higher boundary have membership degree as 0 and centre $b$ has a membership degree of 1 .

Fuzzy Rules: The fuzzy classifier usually operates based on the set of rules that are formulated based on the parameter fuzzy values. In our proposed system, the fuzzy rules are formulated based on the feature vector values and these rules are further subjected to optimization for better classification. We have utilized AGA for optimizing the rules. In our proposed method, the Defuzzification step is avoided and the rules obtained after the fuzzy 
process is subjected to optimization which will further refine the classification process.

\subsubsection{Rule Optimization using AGA}

Adaptive genetic algorithm is an improved version of genetic algorithm where we utilize adaptive mutation for bettering the optimization result. In a normal genetic algorithm, the mutation is employed to each parent chromosome where it interchanges the genes randomly. In our proposed Adaptive mutation, a mutation rate is calculated based on the fitness of the chromosome. The mutation is performed based on this mutation rate instead of random interchange. For AGA to function, we have to generate chromosomes for the solution set. Each chromosome will be subjected to various steps in AGA.

\subsubsection{Various Steps Using Adaptive Genetic Algorithm}

The adaptive genetic algorithm usually consists of five steps namely

- Chromosome Generation

- Calculating Fitness function

- Crossover

- Adaptive Mutation

- Selection

For optimizing the rules generated by the fuzzy classifier, we consider each rule as chromosome. The pools of chromosome are generated randomly and each chromosome is subjected to various operations in AGA. The Chromosomes are evaluated based on the fitness value and those chromosomes with better fitness value are available at the output.

\section{Step 1: Chromosome Generation}

The initial stage in the adaptive genetic neural network is the generation of chromosomes. Here the chromosomes are the number of rules generated using fuzzy and the genes are the rule parameters. In the solution space, we generate ' $C$ ' numbers of random chromosomes which is shown in below expression

$$
\begin{aligned}
C h_{k} & =\left[G_{0}{ }^{k} G_{1}{ }^{k} \ldots G_{C_{L-1}}{ }^{k}\right] \\
0 & \leq k \leq M-1 ; 0 \leq i \leq C_{L}-1
\end{aligned}
$$

Where $G_{i}{ }^{k}-j^{t h}$ gene of chromosome

$M$ - Total population

$C_{L^{-}}$Length of chromosome

\section{Step 2: Calculating Fitness function}

The fitness function is obtained using the expression given in eq. 11. The fitness function is the major parameter in an optimization algorithm for selection of solutions. Those solutions which possess better fitness will be selected for further proceedings.

$$
f t=\sum_{k=1}^{M} R_{s} / M
$$

Where $R_{s}$ - selected rule; $M$ - total number of rules

The fitness value for every chromosome is calculated based on the rules being selected. These fitness values are the initial fitness for each chromosome and are then compared with the fitness of newly generated chromosome for selecting the best solutions.

Step 3: Crossover

In order to generate a new chromosome, crossover operation is performed between two parent chromosomes. The new chromosome thus generated is called offspring. The crossover is done based on selected genes and offspring are produced based on the crossover rate $C O_{\text {rate }}$. The expression for finding out the cross over point is given in eq. 12

$$
\mathrm{CO}_{\text {rate }}=\frac{C_{G}}{C_{L}}
$$

Where $\mathrm{CO}_{\text {rate }}$ - Crossover rate

$C_{G}$ - Number of gene crossovered.

$C_{L}$ - Length of chromosome.

Based on the crossover rate calculated using the above expression, the parent chromosomes are crossovered to generate a new set of chromosome called offspring. By utilizing the cross over rate, the crossover point is found out and the genes at these points are interchanged from both the parent chromosome in order to generate the offspring which contains the characteristics of both the parent chromosome. These chromosomes attain better fitness when compared to the old generation of chromosome thereby making it better for processing.

\section{Step 4: Adaptive Mutation}

The proposed technique utilized adaptive mutation instead of normal mutation step which involves interchange of genes from single parent chromosome. In our proposed adaptive mutation, the mutation is carried out based on the mutation rate. The mutation is performed for each chromosome based on the mutation rate calculated. The expression for estimating the mutation rate is given in eq. 13 below.

$$
\begin{array}{ll} 
& M U_{r}=\frac{P_{m}}{C_{L}} \\
\text { Where } \quad & M U_{r} \text { - mutation rate } \\
& P_{m} \text { - mutation point. } \\
& C_{L} \text { - length of chromosome. }
\end{array}
$$

The mutation rate is selected based on the fitness value that we estimate. The fitness value that we utilize here is based on the rules we generate using fuzzy logic. The mutation rate is then compared with these fitness values and the resultant values 
corresponding to the threshold are chosen as the final mutation rate. The vector that represents the possible mutation points is given as follows

$$
M U_{r}=\left\{m p_{1}, m p_{2} \ldots m p_{l}\right\}
$$

Here ' $l$ ' represents the chromosome length. The mutation rate will be identified based on the $f t$.

$$
M U_{r}=\left\{\begin{array}{c}
1 ; \text { ifft } \leq=T \\
0 ; \text { else }
\end{array}\right.
$$

Here ' $T$ ' is calculated based on the fuzzy rule generated. The mutation is performed for each mutation point extracted using eq. 14. The mutation rate varies for each chromosome in each iteration depending on the fitness value.

\section{Step 5: Selection}

The final stage in adaptive genetic algorithm is the selection process. Based on the obtained fitness value, the new chromosomes $\left(N_{p}\right)$ are positioned in a selection pool. The chromosomes with best fitness value remains in the top of the selection pool. The top most $N_{p}$ chromosomes stored in selection pool are chosen as the next generation between the $2 N_{p}$ chromosomes. The major consideration for selection is the fitness value of a particular chromosome.

\section{Results and discussion}

The proposed Sign Gestures Language Data Acquisition and Recognition is implemented in the working platform of MATLAB. The proposed technique of gesture recognition is examined with frames extracted from different videos. Each frame is subjected to segmentation which is followed by feature extraction and classification in order to recognize the gestures. The obtained results of the proposed system are shown in figures below.

Each segmented input frame is subjected to feature extraction and these features help in identifying the gestures in each frame. Once the features are extracted then the exact words are recognized. Fig 2 and 3 shows the results obtained by the proposed AGFC method.

Each image delivers different words as sign gesture and these images after processing through the different steps in our proposed method and finally the gesture is recognized. Fig 4 shows the recognized words from different inputs by the AGFC method.

The average recognition rate is considered as the main parameter in our proposed technique for evaluating the performance along with the mutation rate. The sign gestures are extracted with the Gabor functions which are discussed in the mathematical equations $7,8,9$ respectively. The recognition rate for our proposed Adaptive Genetic Fuzzy Classifier is compared with that of existing classifiers (Neural Networks).

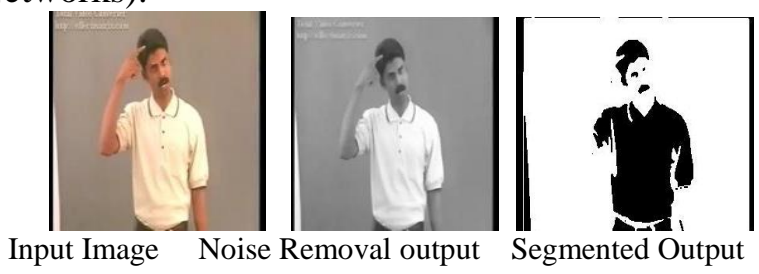

Figure.2 Sign Gesture Recognition for 'Black'
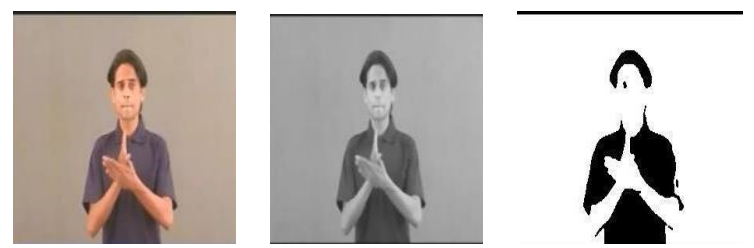

Input Image Noise Removal output Segmented Output

Figure.3 Sign Gesture Recognition for 'Meat'

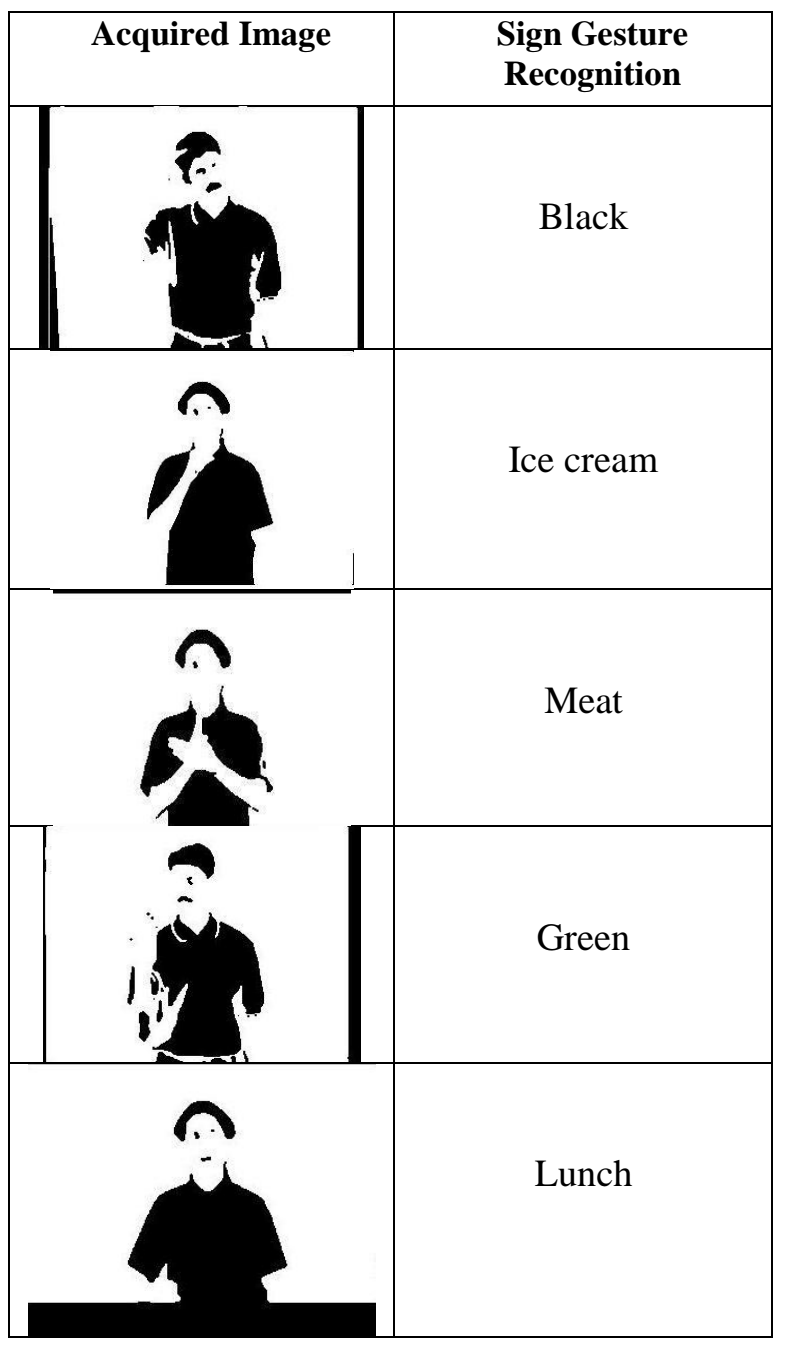

Figure.4 Classification of various Sign Gestures 
Table 1. Recognition rate for proposed Adaptive Genetic Fuzzy Classifier method and existing neural network method.

\begin{tabular}{|c|c|}
\hline Method & $\begin{array}{c}\text { Average Recognition } \\
\text { Rate (\%) }\end{array}$ \\
\hline $\begin{array}{c}\text { Adaptive Genetic } \\
\text { Fuzzy classifier } \\
\text { (Proposed method) }\end{array}$ & 83 \\
\hline $\begin{array}{c}\text { Neural Network } \\
\text { (Existing method) }\end{array}$ & 79 \\
\hline
\end{tabular}

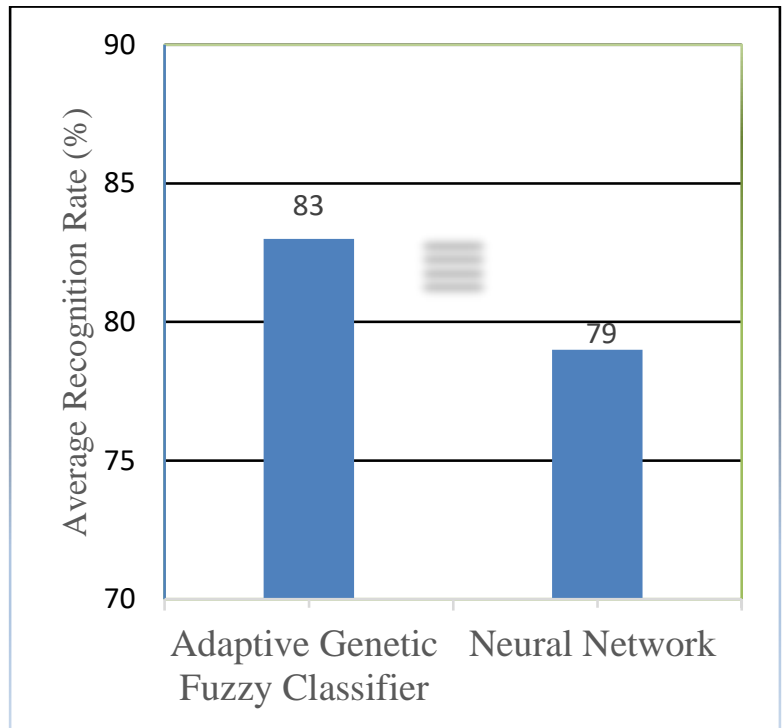

Figure.5 Recognition rate for proposed Adaptive Genetic Fuzzy Classifier method and existing neural network method.

Finally, the accuracy of the proposed recognition rate is compared against the work in [23, 24]. The work in [23] used Neural Network Classifier (NNC) and obtained $79 \%$ average recognition rate for static gestures. However, using modified region growing algorithm, AGFC and the proposed average recognition rate is increased to $83 \%$. This is a clear advantage and well justified with the various gestures which are shown in the below figures.

The existing classifier which we compare here is the neural network [23]. The result obtained shows that our proposed system has a better recognition rate when compared to existing system. The below table shows the recognition rate obtained for proposed Adaptive Genetic Fuzzy Classifier method and existing neural network method.

From the table 1, we can infer that the average recognition rate using proposed AGFS classifier is better than that of existing neural network classifier. The corresponding graphical representation of the recognition rate is shown in the fig 5. Thus, the proposed system of sign gesture classification using adaptive genetic fuzzy classifier has proved to be more efficient in terms of recognition of words from sign gesture thereby improving the communication between deaf and normal persons.

\section{Conclusion}

In this work, we have developed a proficient technique for Sign gesture acquisition and recognition with the aid of modified region growing algorithm and AGFS. The sign gesture forms a basic mode of communication with the deaf community and its acquisition and recognition plays a vital role for the communication with those who are unfamiliar with sign gestures. Our proposed method helps in recognizing each and every gesture with higher accuracy and less effort. We employed feature extraction stage along with the segmentation which forms an efficient process in extracting the required measures for recognition. This is followed by an AGFS based classification process which delivers the exact recognized output from the sign gesture. The results of our method show that it is more efficient when we compare with other existing works related to sign gesture recognition. In future, we plan to work for few more gestures especially for the gestures of children.

\section{Reference}

[1] C. Zhu and W. Sheng, "Wearable sensor- based hand gesture and daily activity recognition for robotassisted living”, IEEE Transactions on Systems, Man, and Cybernetics-Part A: Systems and Humans, 41(3), pp.569-573, 2011.

[2] T. Zhang and Z. Feng, "Dynamic Gesture Recognition Based on Fusing Frame Images", In Intelligent Systems Design and Engineering Applications, Fourth International Conference pp.280-283, 2013.

[3] G. Fang, W. Gao and D. Zhao "Large vocabulary sign language recognition based on fuzzy decision trees", IEEE Transactions on Systems, Man, and Cybernetics-Part A: Systems and Humans, 34(3), pp.305-314, 2004.

[4] R. Xu, S. Zhou and W. J. Li, "MEMS accelerometer based nonspecific-user hand gesture recognition", IEEE sensors journal, 12(5), pp.11661173, 2012.

[5] J. S. Wang and F. Chuang, "An accelerometer-based digital pen with a trajectory recognition algorithm for handwritten digit and gesture recognition", IEEE Transactions on Industrial Electronics, 59(7), pp.2998-3007, 2012.

[6] C. Tran and M.M. Trivedi, "3-D posture and gesture recognition for interactivity in smart spaces". IEEE 
Transactions on Industrial Informatics, 8(1), pp.178$187,2012$.

[7] U. Von Agris, M. Knorr and K.F. Kraiss, "The significance of facial features for automatic sign language recognition", In Automatic Face \& Gesture Recognition, 2008. FG'08. 8th IEEE International Conference on pp.1-6, 2008.

[8] R. Yan, K. Tee, Y. Chua and H. Tang, "Gesture recognition based on localist attractor networks with application to robot control" [application notes]. IEEE Computational Intelligence Magazine, 7(1), pp.64-74, 2012.

[9] R. Kaluri, Ch.P. Reddy, "An Overview on Human Gesture Recognition", International Journal of PT, 8(2), pp.12037-12045, 2016.

[10] Z. Ren, J. Yuan, J. Meng and Z. Zhang, "Robust part-based hand gesture recognition using Kinect sensor", IEEE transactions on multimedia, 15(5), pp.1110-1120, 2013.

[11] H.D. Yang, S. Sclaroff and S.W. Lee, "Sign language spotting with a threshold model based on conditional random fields", IEEE Transactions on Pattern Analysis and Machine Intelligence, 31(7), pp.1264-1277, 2009.

[12] S. Mitra and T. Acharya, "Gesture recognition: A survey", IEEE Transactions on Systems, Man, and Cybernetics, Part C (Applications and Reviews), 37(3), pp.311-324, 2007.

[13] D. Frolova, H. Stern and S. Berman, "Most probable longest common subsequence for recognition of gesture character input", IEEE transactions on cybernetics, 43(3), pp.871-880, 2013.

[14] B. Wang, X. Chang, X and C. Liu, "Skin detection and segmentation of human face in color images", International Journal of Intelligent Engineering and Systems, 4(1), pp.10-17, 2011.

[15] H. Cheng, J. Luo and X. Chen, "A windowed dynamic time warping approach for $3 \mathrm{D}$ continuous hand gesture recognition", In 2014 IEEE, International Conference on Multimedia and Expo (ICME) pp. 1-6, 2014.

[16] A. Akl, C. Feng and S. Valaee, "A novel accelerometer-based gesture recognition system", IEEE Transactions on Signal Processing, 59(12), pp.6197-6205, 2011.

[17] V. Kosmidou, C. Petrantonakis and L.J. Hadjileontiadis, "Enhanced Sign Language Recognition Using Weighted Intrinsic-Mode Entropy and Signer's Level of Deafness". IEEE Transactions on Systems, Man, and Cybernetics, Part B (Cybernetics), 41(6), pp.1531-1543, 2011.

[18] V. Kosmidou and L.J. Hadjileontiadis, "Sign language recognition using intrinsic-mode sample entropy on sEMG and accelerometer data", IEEE transactions on biomedical engineering, 56(12), pp.2879-2890, 2009.

[19] N. Tubaiz, T. Shanableh and K. Assaleh, "Glovebased continuous Arabic sign language recognition in user-dependent mode", IEEE Transactions on Human-Machine Systems, 45(4), pp.526-533, 2015.
[20] Y. Yao and Y. Fu, "Contour model-based handgesture recognition using the Kinect sensor", IEEE Transactions on Circuits and Systems for Video Technology, 24(11), pp.1935-1944, 2014.

[21] R. Yang, S. Sarkar and B. Loeding, "Handling movement epenthesis and hand segmentation ambiguities in continuous sign language recognition using nested dynamic programming", IEEE transactions on pattern analysis and machine intelligence, 32(3), pp.462-477, 2010.

[22] T.H.S. Li, M.C. Kao and P.H. Kuo, P. H, "Recognition System for Home-Service-Related Sign Language Using Entropy-Based-Means Algorithm and ABC-Based HMM", IEEE Transactions on Systems, Man, and Cybernetics: Systems, 46(1), pp.150-162, 2016.

[23] T. Ahmed, "A neural network based real time hand gesture recognition system", International journal of computer applications, 59(4), pp.17-22, 2012.

[24] R. Kaluri and Ch. P. Reddy, "A framework for sign gesture recognition using improved genetic algorithm and adaptive filter. Cogent Engineering, 2016 (accepted).

[25] K. Lakshmanna, N. Khare. "Constraint-Based Measures for DNA Sequence Mining using Group Search Optimization Algorithm." International Journal of Intelligent Engineering and systems 9(3) pp.91-100, 2016.

[26] G.T. Reddy, N. Khare, "FFBAT-Optimized Rule Based Fuzzy Logic Classifier for Diabetes", International Journal of Engineering Research in Africa, 24(2), pp.137-152, 2016. 